\title{
Comparison of Different Simulation Approaches in Ring-Core Method
}

\author{
František Menda ${ }^{1, *}$, Patrik Šarga ${ }^{2}$, Tomáš Lipták ${ }^{2}$, František Trebuňa ${ }^{1}$ \\ ${ }^{1}$ Technical University of Košice, Faculty of Mechanical Engineering, Department of Applied Mechanics and Mechanical Engineering, \\ Košice, Slovakia \\ ${ }^{2}$ Technical University of Košice, Faculty of Mechanical Engineering, Department of Mechatronics, Košice, Slovakia \\ *Corresponding author: frantisek.menda@tuke.sk
}

Received October 08, 2014; Revised October 15, 2014; Accepted November 06, 2014

\begin{abstract}
Ring-Core, a semi-destructive method enables measurement of the residual stresses inside the material by the sensor attached at the top of the specimen. Such stress reconstruction requires proper determination of the calculation coefficients. Thus the simulation model for finite elements analyzes is created by means of two different commercial software. Subsequently both simulation results are compared experimentally by measurement on the specimen loaded by known uniaxial state of stress.
\end{abstract}

Keywords: residual stress, Ring-Core, SolidWorks, ANSYS, FEM, incremental method, differential method

Cite This Article: František Menda, Patrik Šarga, Tomáš Lipták, and František Trebuňa, "Comparison of Different Simulation Approaches in Ring-Core Method.” American Journal of Mechanical Engineering, vol. 2, no. 7 (2014): 258-261. doi: 10.12691/ajme-2-7-17.

\section{Introduction}

Residual stress prediction in steel constructions is important task for life and safety prediction of the components. In last 20 years major changes have been done in the field of experimental measurement as well as in simulation procedures. Ring-Core method, a semidestructive method is now relatively common due to the sufficient commercial measuring equipment. However the connections between experimental measurement and simulation studies is still not sufficient which is showed by lack of some international standard. Prerequisite for experimental usage of the Ring-Core method for evaluation of the residual stress components in material is the determination of adequate calculation coefficients and constants $[1,2,3]$. Sensing of the stress changes inside the material by the sensor attached at the top of the component is not suitable by using the classic Hook's law. Thus, there are three commonly used evaluation techniques for residual stress determination: incremental, differential and integral. All of them are derived from the Hook's law and also from ASTM E837-13a [4] adapted to the Ring-Core method. Although these methods are well known and used, there is still no unique set of calculation coefficients covering the geometric parameters of the tested component.

Appropriate simulation model for finite elements method calculations is the most effective way for determination of the right calculation parameters. Two different approaches for this task in connection with software ANSYS and SolidWorks were used in this contribution. Simulation results were also confronted to those acquired by experimental measurement carried out by commercially available measuring equipment.

\section{Simulation Model in SolidWorks}

From published works it is known that only one universal set of calculation coefficients is needed, which is unique for specific material parameters, type of strain gage rosette and the parameters of created notch, to determine the direction, sense and magnitude of the residual stress components inside the component [1,4,5,6,7]. Thus the simple model of a cube with sufficiently large dimensions to avoid unwanted boundary influences on calculation accuracy was chosen as a simulation model. Quicker simulation analyzes were achieved by means of quarter model of a cube. The dimensions of a model were: $50 \mathrm{~mm} \times 50 \mathrm{~mm}$ and the thickness $100 \mathrm{~mm}$. Figure 1 shows created simulation model in software SolidWorks. It includes Ring-Core notch with the standard parameters: $14 \mathrm{~mm}$ inner and $18 \mathrm{~mm}$ outer diameter with the maximum milling depth $5 \mathrm{~mm}$. Simulated measuring grids represent the parameters of a strain gage rosette XY51, suitable for measuring residual stress with the known principal directions of the components.

Ideal shape of the quarter model enables to use symmetry fixtures applied on two faces close to the measuring grids. This type of fixtures prevents the model movement in the plane parallel with the measuring grids $(x y)$. The last degree of freedom is removed by fixing the corner $A$ of the model.

The finite elements topology of the model consists of the tetrahedral elements and is divided into four areas (see Figure 2). Basic mesh of the model (No.1) is created by 
coarse mesh with size of the elements equals to $6-10 \mathrm{~mm}$. Notch (No.2) is filled with the elements of a size $1 \mathrm{~mm}$, the area of isolated core (No.3) contains elements with the size $0,25 \mathrm{~mm}$. Measuring grids, stand-alone components are meshed according the parameters of strain gage rosette XY51 by the elements with the size $0,0114 \mathrm{~mm}$.

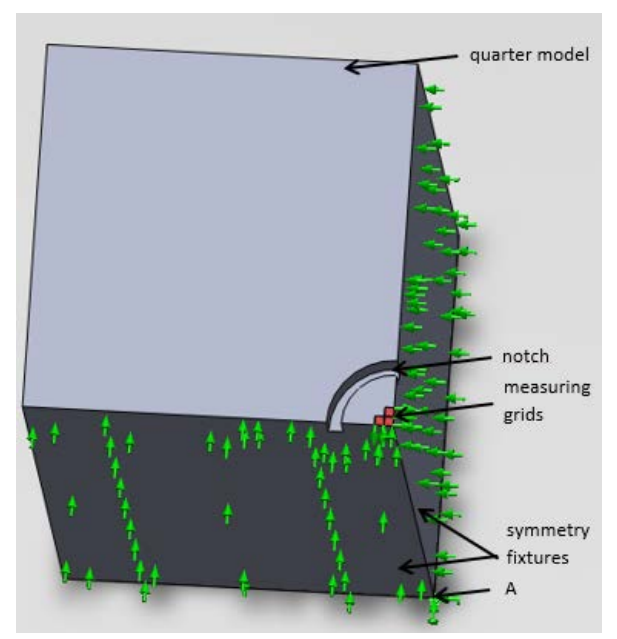

Figure 1. Quarter model in SolidWorks

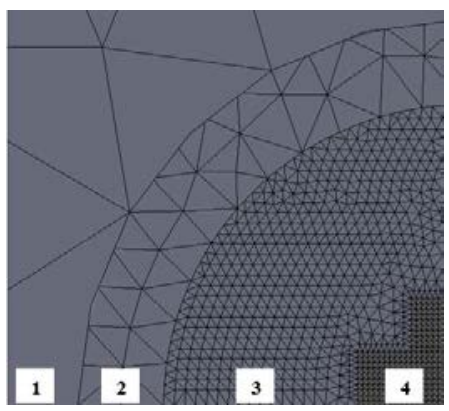

Figure 2. Close up of the mesh topology of SolidWorks model

\section{Simulation Model in ANSYS}

Simulation model created in software ANSYS was used to achieve the best accuracy of the acquired calculation coefficients. ANSYS enables to use hexahedron element type with 20 nodes. However the mesh topology is more complicated and requires complex approach. The model was created (Figure $3 a$ ) and subsequently meshed (Figure 3b) from the finest parts for relieved strain sensing, through the part of isolated core, annular notch, crossing part, up to the part of the model filled with the coarsest elements.

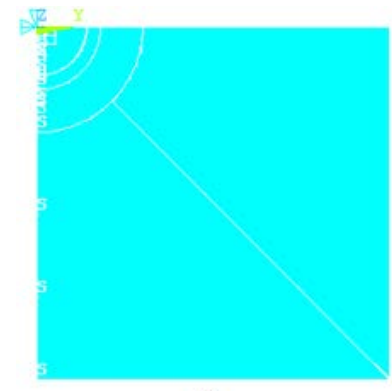

a)

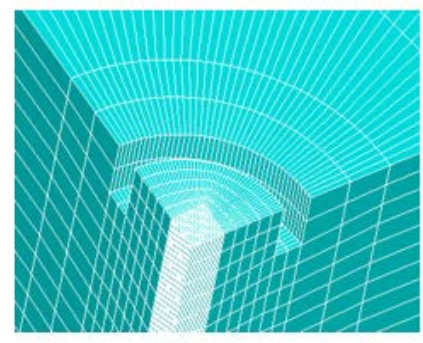

b)
Figure 3. a)model topology, b)close up of the mesh topology

Simulation model was fixed similarly to the one created in SolidWorks.

\section{Principle of Simulation Procedure}

Residual stress inside the model cannot be simulated directly, the object has to be activated by the known stress field which causes the same deformation changes of the model as residual stress. Activation of outer faces of the model was chosen in case of study incremental and differential evaluation methods. Subsequently the strain data acquisition was performed by the integration through the area of simulated measuring grids. Strains in principal directions $(x, y)$ measured before the notch creation were marked as reference $\left(\varepsilon_{10}, \varepsilon_{20}\right)$. After each milled depth $z$ the relieved strains $\varepsilon_{1,2 m}(z)$ were measured and the strain values corresponding to the strain gage measurement were calculated:

$$
\varepsilon_{1,2}(z)=\varepsilon_{1,2 m}(z)-\varepsilon_{10,20}
$$

For all simulation studies in this work the used material was linear, elastic, isotropic with the Young's modulus $E=210 G P a$ and Poisson's number $\mu=0$,3. Iterative solver Pre-Conditioned Conjugate Gradient was chosen in ANSYS and direct sparse in SolidWorks.

\subsection{Incremental Method}

Incremental evaluation method is based on a principle that each increment of a relieved strain $d \varepsilon$ measured at the top of the component after milled depth increment $d z$ is fully caused by the stress acting at that very increment. Stresses that act in previous steps, respectively stresses perpendicular to the measuring grids are negligible to those measured in the plane of the grids. Thus the method is suitable for the evaluation of residual stress uniformly distributed through the model thickness. The strain gage sensitivity to the inner stress is determined by the calibration coefficients $K_{1}, K_{2}$ calculated by equations (2)(5) $[1,8]$.

$$
\begin{gathered}
\frac{d \varepsilon_{1}}{d z}=\varepsilon_{1}^{\prime} \\
\frac{d \varepsilon_{2}}{d z}=\varepsilon_{2}^{\prime} \\
K_{1}=\frac{E}{\sigma_{1}} \cdot \varepsilon_{1}^{\prime} \\
K_{2}=-\frac{E}{\mu \sigma_{1}} \cdot \varepsilon_{2}^{\prime}
\end{gathered}
$$

\subsection{Differential Method}

Differential method represents the special type of the incremental method. The magnitude of the residual stress components is calculated just in two steps $z_{i}$ and $2 z_{i}$. Firstly the strain differences are calculated:

$$
\begin{aligned}
& \Delta \varepsilon_{1}=\left(\varepsilon_{1}\right)_{2 z_{i}}-\left(\varepsilon_{1}\right)_{z_{i}} \\
& \Delta \varepsilon_{2}=\left(\varepsilon_{2}\right)_{2 z_{i}}-\left(\varepsilon_{2}\right)_{z_{i}}
\end{aligned}
$$

Residual stresses are calculated by means of adequate relaxation coefficients $A$ and $B$ according equations (8) and (9).

$$
\sigma_{1}=A \cdot \Delta \varepsilon_{1}+B \cdot \Delta \varepsilon_{2}
$$




$$
\sigma_{2}=A \cdot \Delta \varepsilon_{2}+B \cdot \Delta \varepsilon_{1}
$$

\section{Comparison}

Comparison of two different simulation approaches of the Ring-Core method is based on the comparison of the calculation coefficients which are necessary for accurate experimental testing.

Firstly the coefficients $K_{1}, K_{2}$ of the incremental method were compared. As it is obvious from Figure 4 the deviations between the calibration coefficients determined by ANSYS and SolidWorks are minimal.

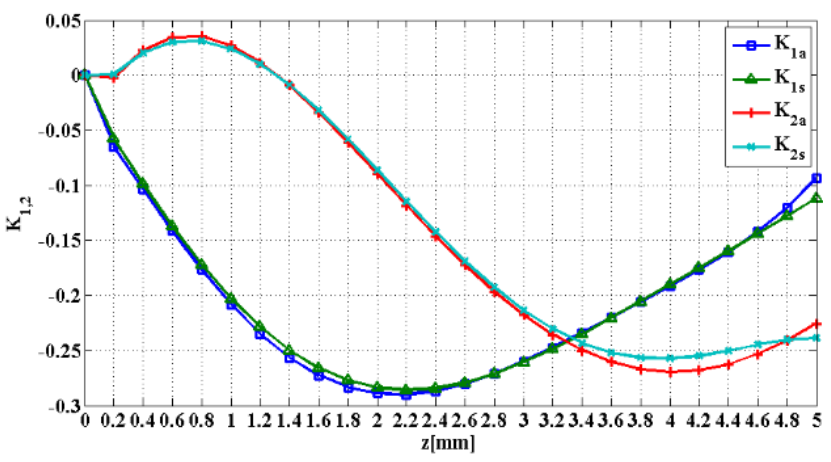

Figure 4. Comparison of calibration coefficients $\mathrm{K}_{1}, \mathrm{~K}_{2}$

Slightly bigger deviations are at the deepest depths which could be caused by the Solidworks mesh topology which was applied on the top of the model, not on the component volumes as it is possible in ANSYS.

Determined relaxation coefficients of the differential method are also very similar in both approaches. In Table 1 the difference $(D A)$ between the relaxation coefficients determined by ANSYS (Aa respectively $B a$ ) and those by SolidWorks (Asrespectively $B s$ ) is $2,4 \%$ respectively $0,1 \%$ for the evaluation depths $z_{i}=1 \mathrm{~mm}$ and $2 z_{i}=2 \mathrm{~mm}$ and $-0,1 \%$ respectively $-2,8 \%$ for the most relevant evaluation depths $z_{i}=2 \mathrm{~mm}$ and $2 z_{i}=4 \mathrm{~mm}$.

Table 1. Comparison of relaxation coefficients $A, B$

\begin{tabular}{|c|c|c|c|c|c|c|}
\hline & \multicolumn{2}{|c|}{ ANSYS } & \multicolumn{2}{|c|}{ SolidWorks } & \multicolumn{2}{|c|}{ Difference } \\
\hline $\begin{array}{c}\mathrm{Z}_{\mathrm{i}} \\
{[\mathrm{mm}]}\end{array}$ & $\begin{array}{c}-\mathrm{Aa} \\
{[\mathrm{MPa}]}\end{array}$ & $\begin{array}{c}-\mathrm{Ba} \\
{[\mathrm{MPa}]}\end{array}$ & $\begin{array}{c}-\mathrm{As} \\
{[\mathrm{MPa}]}\end{array}$ & $\begin{array}{c}-\mathrm{Bs} \\
{[\mathrm{MPa}]}\end{array}$ & $\begin{array}{l}\text { DA } \\
{[\%]}\end{array}$ & $\begin{array}{l}\mathrm{DB} \\
{[\%]}\end{array}$ \\
\hline 1 & \multirow{2}{*}{786892} & \multirow{2}{*}{32355} & \multirow{2}{*}{805988} & \multirow{2}{*}{32402} & \multirow{2}{*}{2,4} & \multirow{2}{*}{0,1} \\
\hline 2 & & & & & & \\
\hline 2 & \multirow{2}{*}{451975} & \multirow{2}{*}{116321} & \multirow{2}{*}{451455} & \multirow{2}{*}{113092} & \multirow{2}{*}{$-0,1$} & \multirow{2}{*}{$-2,8$} \\
\hline 4 & & & & & & \\
\hline
\end{tabular}

\subsection{Comparison by Means of Experimental Testing}

Assumptions acquired by the simulation procedures were verified also experimentally. Planar specimen was created for this purpose. Firstly the specimen was annealed to ensure no or almost no residual stress inside the material. After the surface preparation and application of the strain gage rosette the measuring string was created (Figure 5) [9] and subsequently the specimen was loaded by the known uniaxial state of stress $\left(\sigma_{1}=60 \mathrm{MPa}\right.$, $\left.\sigma_{2}=0 M P a\right)$.

An automated system MTS 3000 Ring-Core from SINT Technology [3] was used for the notch creation and for the strain data acquisition.

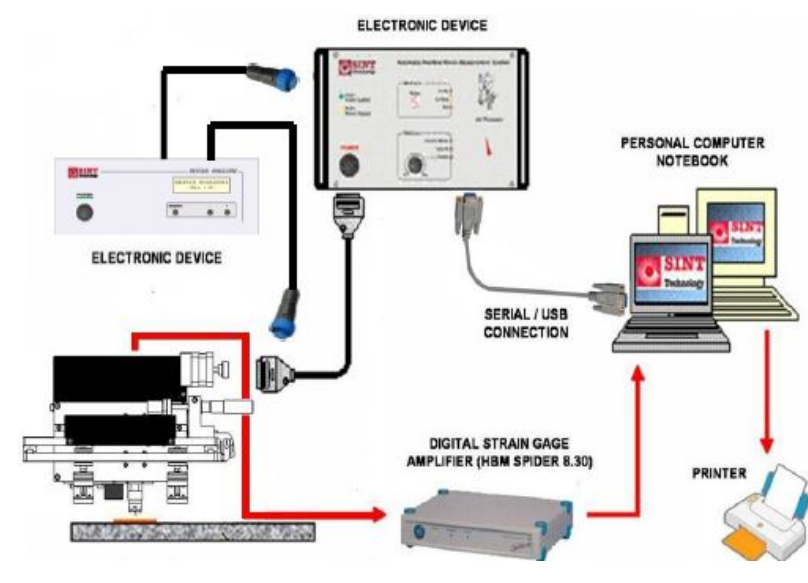

Figure 5. Measuring string

Residual stress evaluation was firstly performed by the incremental method. Both sets of calibration coefficients $K_{1}$ and $K_{2}$ were used for evaluation of the principal residual stress $\sigma_{1}$. Figure 6 shows the deviations of the evaluated residual stresses from the assumed load stress in the dependence of the milled depth $z$. Values calculated by means of calibration coefficients determined by ANSYS are very close to those ones calculated by the calibration coefficients determined by SolidWorks. The deviations at each step between these two approaches are less than $3 \%$, only in first and last the deviations are bigger which is caused by different mesh topology.

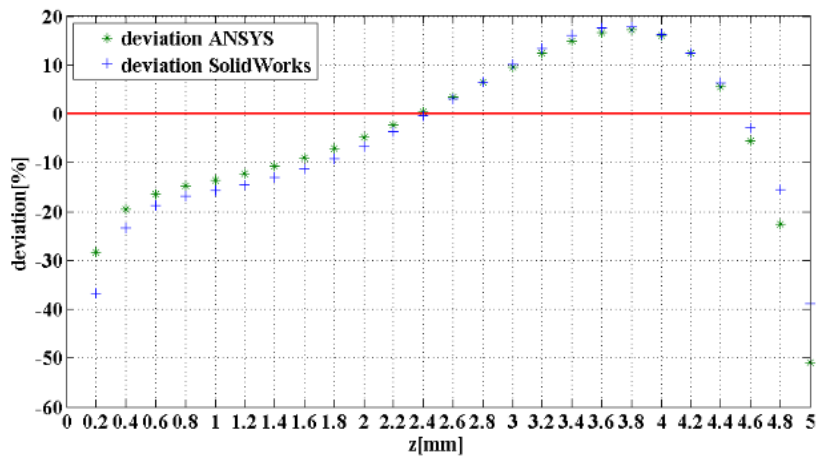

Figure 6. Comparison of two different simulation approaches by the incremental evaluation method

The difference between two simulation approaches was also compared by means of differential evaluation method. Due to the uniaxial state of stress only $A$ relaxation coefficients were compared. Table 2 shows the difference between the reference load and the residual stress evaluated by the ANSYS coefficients $(D a)$ respectively Solidworks relaxation coefficients (Ds). The difference between two approaches is $2,17 \%$ in case of $z_{i}=1 \mathrm{~mm}$ and $2 z_{i}=2 \mathrm{~mm}$ respectively $-0,05 \%$ in case of $z_{i}=2 \mathrm{~mm}$ and $2 \mathrm{z}_{\mathrm{i}}=4 \mathrm{~mm}$.

Table 2. Comparison of two different simulation approachesby the differential evaluation method

\begin{tabular}{|c|c|c|c|c|}
\hline & reference & \multicolumn{2}{|c|}{ difference } & $\begin{array}{l}\text { difference } \\
\text { between }\end{array}$ \\
\hline $\mathrm{z}_{\mathrm{i}}[\mathrm{mm}]$ & $\sigma_{1}[\mathrm{MPa}]$ & $\mathrm{Da}[\%]$ & Ds[\%] & $\mathrm{DA}[\%]$ \\
\hline 1 & \multirow{2}{*}{60} & \multirow{2}{*}{$-10,79$} & \multirow{2}{*}{$-8,62$} & \multirow{2}{*}{2,7} \\
\hline 2 & & & & \\
\hline 2 & \multirow{2}{*}{60} & \multirow{2}{*}{8,33} & \multirow{2}{*}{8,38} & \multirow{2}{*}{$-0,05$} \\
\hline 4 & & & & \\
\hline
\end{tabular}


Experimental testing confirmed the minimal differences between the residual stress evaluation by calculation coefficients determined by ANSYS and by SolidWorks in case of uniform uniaxial residual stress distribution evaluated by the incremental and differential method.

\section{Discussion}

ANSYS and SolidWorks, two commercially available software could be used for Ring-Core research with the similar accuracy. These are some useful notes from own experience:

SolidWorks:

- offers limited mesh options and mesh topology only for the surface,

- strain gage has to be created as separate component,

- mesh creation is independent from the model creation process,

- interface is very friendly,

- parametric simulations are performed very simply and quickly,

- possible scripts using Visual Basic or C\# and sufficient connection with excel workbook[10];

ANSYS:

- great choice of finite elements types and mesh parameters,

- model has to be created according desired mesh topology,

- less friendly interface,

- possible parametric simulations,

- more sensitive to random user mistakes,

- little changes in model geometry require completely new model and mesh topology,

- software tends to crash more times than Solidworks;

\section{Conclusion}

Ring-Core method as a semi-destructive method is based on proper determination of the calculation coefficients. For this task two different simulation approaches were used by commercially available software ANSYS and SolidWorks. Relative simplicity of the chosen simulation model causes the minimal differences between the results obtained from both programs although different finite elements were used for mesh creation. Negligible differences in determined calculation coefficients enable to use both calibration sets in experimental measurements and evaluations by means of incremental and differential method without losing the accuracy. The development of the Ring-Core method continues by close connection of simulation analyzes performed by finite elements method with experimental verification in laboratory conditions. Also the comparison of both simulation approaches in case of non-uniform stress distribution through the material thickness is the matter of our research.

\section{Acknowledgement}

The authors would like to thank to Slovak Grant Agency - project VEGA 1/0937/12 "Development of unconventional experimental methods for mechanic and mechatronic systems” and project FGV/2013/9 “Complex analysis of the Ring-Core method in order to create the evaluation program”.

\section{References}

[1] F. Menda, F. Trebuňa, P. Šarga: Determination of the Necessary Geometric Parameters of the Specimen in Ring-Core Method. Applied Mechanics and Materials, 2014, 486, p. 90. ISSN: 16627482.

[2] F. Menda, F. Trebuňa, P. Šarga: Estimation of residual stress field uniformity when using the Ring-Core method. Advanced Materials Research Vol. 996 (2014) pp 325-330.

[3] E. Valentini, A. Benincasa, L. Bertelli: An automatic system for measuring residual stresses by Ring-core method. Italian Stress Analysis Association, 40th National convention, University of Palermo, 2011.

[4] ASTM International Designation E 837-13a: Standard Test Method for Determining Residual Stresses by the Hole-Drilling Strain Gauge Method, United States, 2013, 16 p.

[5] B. Zuccarello: Optimization of Depth Increment Distribution in the Ring-Core Method. Journal of Strain Analysis for Engineering Design. July 1996, Vol. 31, no. 4, p. 251-258.

[6] F. Trebuňa, F. Šimčák, Handbook on experimental mechanics (In Slovak "Príručka experimentálnej mechaniky"), first ed., Košice SjF TU, 2007, 1526 p.

[7] M.Barsanti et al., Integral method coefficients and regularization procedure for the ring-core residual stress measurement technique. Advanced Materials Research Vol. 996(2014) pp 331-336.

[8] D. von Mirbach: Hole-Drilling Method for Residual Stress Measurement- Consideration of Elastic-Plastic Material Properties. Materials Science Forum, vol. 768-769, pp. 174-181, 2013.

[9] P. Šarga, F. Menda, Analysis of Measuring Chain for Evaluating Residual Stresses by Ring-Core Method, In: American Journal of Mechanical Engineering, Vol. 1, no. 7 (2013), p. 313-317.

[10] F. Menda et al., SolidWorks API for Ring-Core Simulations, In: SAMI 2014: IEEE 12th International Symposium on Applied Machine Intelligence and Informatics: proceedings: January 23-25, 2014, Herl'any, Slovakia. 